\title{
Continuous Positive Airway Pressure Treatment for Obstructive Sleep Apnea Does Not Reduce Arterial Stiffness in Patients With Type 2 Diabetes After One Year of Follow-Up
}

\author{
Christoffer Krogager a, b, c, d, i, Anne Margareta Banghoje, Per L. Poulsen ${ }^{\mathrm{c}}$, \\ Martin G. Kirkegaard ${ }^{f}$, Birger Thorsteinsson ${ }^{\mathrm{e}} \mathrm{g}$, Lise Tarnow ${ }^{\mathrm{e}}$, h, \\ Esben Laugesen ${ }^{\mathrm{c}}$, Klavs W. Hansen ${ }^{\mathrm{b}}$
}

\begin{abstract}
Background: The aim of this study is to evaluate the effects of 12-month continuous positive airway pressure (CPAP) treatment on arterial stiffness in patients with type 2 diabetes.

Methods: Obstructive sleep apnea (OSA) and type 2 diabetes frequently co-exists. Both diseases increase arterial stiffness, a marker of cardiovascular risk. Treating OSA with CPAP may lower arterial stiffness. In a recent randomized trial, we found that CPAP treatment for 12 weeks did not reduce arterial stiffness in type 2 diabetes patients with OSA. Participants from the randomized trial were invited to a follow-up study 12 months after inclusion. We evaluated arterial stiffness by measuring carotid-femoral pulse wave velocity (cfPWV) using SphygmoCor.
\end{abstract}

Results: Forty-six patients $(63.9 \%$ of the original 72 patients, age $63.8 \pm 6.5$ years, diabetes duration $16.1 \pm 9.7$ years, body mass index (BMI) $34.7 \pm 3.9 \mathrm{~kg} / \mathrm{m}^{2}$ ) partook in the study. Mean duration of CPAP treatment was $10.5 \pm 1.5$ months. Baseline cfPWV was $10.7 \mathrm{~m} / \mathrm{s}$. At

Manuscript submitted September 3, 2021, accepted October 4, 2021

Published online October 31, 2021

aDepartment of Clinical Medicine, Aarhus University, Palle Juul-Jensens Blvd. 82, 8200 Aarhus, Denmark

bDiagnostic Centre, Regional Hospital Silkeborg, Falkevej 1A, 8600 Silkeborg, Denmark

'Department of Endocrinology and Internal Medicine, Aarhus University Hospital, Palle Juul-Jensens Blvd. 99, 8200 Aarhus N, Denmark

${ }^{\mathrm{d}}$ The Danish Diabetes Academy, Sondre Blvd. 29, 5000 Odense, Denmark

eDepartment of Cardiology, Nephrology and Endocrinology, Nordsjaellands Hospital, Dyrehavevej 29, 3400 Hillerod, Denmark

${ }^{f}$ Sleep Disorders Clinic, Elective Surgery Centre, Regional Hospital Silkeborg, Falkevej 1A, 8600 Silkeborg, Denmark

gDepartment of Clinical Medicine, Faculty of Health and Medical Sciences, University of Copenhagen, Blegdamsvej 3B, 2200 Kobenhavn N, Denmark

${ }^{h}$ Steno Diabetes Center Zealand, Birkevaenget 3, 3.sal. 4300 Holbaek, Denmark ${ }^{i}$ Corresponding Author: Christoffer Krogager, Department of Endocrinology and Internal Medicine, Aarhus University Hospital, Palle Juul-Jensens Blvd. 99, DK-8200 Aarhus N, Denmark. Email: chikrg@rm.dk follow-up cfPWV was $10.6 \mathrm{~m} / \mathrm{s}$, change in cfPWV: $-0.12 \mathrm{~m} / \mathrm{s}, 95 \%$ confidence interval $(\mathrm{CI})$ : $-0.6,0.4, \mathrm{P}=0.6$. Baseline systolic blood pressure (BP) was $136.2 \mathrm{~mm} \mathrm{Hg}$. At follow-up BP was $137.9 \mathrm{~mm} \mathrm{Hg}$, change in BP: $1.6 \mathrm{~mm} \mathrm{Hg}, 95 \% \mathrm{CI}:-2.3,5.5$.

Conclusions: We found no effect of 9 - 12-month CPAP treatment on arterial stiffness or BP in patients with long duration of type 2 diabetes and OSA.

Keywords: Arterial stiffness; Pulse wave velocity; Diabetes; Obstructive sleep apnea

\section{Introduction}

Obstructive sleep apnea (OSA) is characterized by repeated episodes of partial or complete occlusion of the upper airways resulting in fragmented sleep and intermittent hypoxemia. OSA is highly prevalent in patients with type 2 diabetes [1]. Both OSA and type 2 diabetes are associated with an increase in cardiovascular disease (CVD) and mortality [2-5].

Arterial stiffness is an early marker of cardiovascular (CV) risk and has been shown to predict CV events independently of traditional risk factors $[6,7]$. In addition, reduction in arterial stiffness may be associated with improved CV outcome [8, 9]. The gold standard for assessing arterial stiffness is carotidfemoral pulse wave velocity (cfPWV) [10]. Both type 2 diabetes and OSA are associated with increased arterial stiffness [11-14].

In our recent randomized study [15] the use of continuous positive airway pressure (CPAP) for 3 months did not reduce PWV in patients with type 2 and OSA. After the study period ended, all patients received treatment with CPAP. The aim of this study is to evaluate the effects of CPAP on arterial stiffness at follow-up of 1 year after the original study.

\section{Materials and Methods}

The study design and inclusion criteria have been reported 
in details previously [15]. Briefly, the patients were recruited from outpatient clinics at three Danish hospitals. The patients were screened for OSA using ApneaLink (ResMed Corp., CA, USA).

\section{Subjects}

Subjects with type 2 diabetes were eligible for participating in the study if they had treatment naive OSA with apnea-hypopnea index (AHI) of 15 or more and were 18 years or older. Exclusion criteria included history of stroke or myocardial infarction within 3 month prior to study start; employment in transport industry; uncontrolled hypertension $>160 / 95 \mathrm{~mm}$ $\mathrm{Hg}$, heart failure (New York Heart Association (NYHA) class III or IV); history of alcohol or drug abuse; pregnant or nursing women.

\section{Study design}

The study is a follow-up of a randomized controlled multicentre study with an intervention of 3 months of CPAP treatment versus no treatment. The study was approved by the local research ethics committee and registered at Clinicaltrials.gov under the identifier NCT02482584. The study was conducted according to the Declaration of Helsinki (ethical principles for medical research involving human subjects). After 3 months the patients in the control group were invited to receive CPAP treatment and the patients in the active treatment arm to continue CPAP. All the participants were invited for a follow-up evaluation 12 months after inclusion in the original study and thus had duration of CPAP treatment between 9 and 12 months. As all patients received treatment at follow-up the patients were treated as a single group.

\section{Study parameters}

At baseline and at the follow-up visit blood pressure (BP) was measured using a fully automated oscillometric device (Microlife WatchBP Office, Microlife AG, Widnau, Switzerland). Measurements were performed after a resting period of $10 \mathrm{~min}$ with the patient in a sitting position.

After BP measurement the patients rested for $5 \mathrm{~min}$ in the supine position after which measurements of arterial stiffness were performed. Arterial stiffness was measured as cfPWV using the SphygmoCor device (version 9, AtCor Medical, Sydney, Australia). Using a tonometer (Millar, STP-304, Houston, Texas, USA), sequential electrocardiogram-referenced recordings of the pulse waves at the carotid and femoral arteries were performed. The transit time was determined using the intersecting tangent method [16]. The travel distance of the pulse wave was determined as the distance from the measuring point at the carotid artery to the measurement point in the femoral artery multiplied by 0.8 as recommended [10]. The distance was measured using a caliper. We performed three consecutive measurements. As recommended by the manufacturer, only
cfPWV measurements with a standard deviation of less than $20 \%$ were accepted.

\section{Study endpoints}

The primary endpoint of our study was the change in cfPWV from baseline to 12-month follow-up. Secondary endpoint was change in BP from baseline to 12-month follow-up.

\section{Statistical analysis}

Data distribution was assessed by histograms and QQ-plots. Summary data are presented as frequencies, as mean \pm standard deviation (SD) for normally distributed data or as median and interquartile range for skewed data. Primary outcome analyses were performed using a linear mixed effect model. Statistical analysis was performed using STATA software package version 14.2 (StataCorp, Texas, USA). A two-sided P value of less than 0.05 was considered to be statistically significant.

\section{Results}

Forty-six (63.9\% of the original 72 patients included in the study) accepted our invitation to participate in the follow-up measurements. Twenty-two patients from the treatment group and 24 patients from the control group accepted participation (Table 1).

No differences were observed between the group accepting follow-up at 12 months and the group who refrained other than a higher number of patients using sodium-glucose cotransporter 2 (SGLT2) inhibitors in the group who refrained from participation in the follow-up (Supplementary Material 1, www.jofem.org).

In a multivariate analysis we found that higher age, higher levels of hemoglobin A1c (HbA1c), current smoking and longer diabetes duration was associated with higher baseline cfPWV. Higher levels of creatinine were associated with a lower cfPWV, whereas baseline body mass index (BMI) or AHI had no effect on cfPWV (Table 2).

\section{Use of CPAP}

The mean duration of CPAP use at follow-up was $10.5 \pm 1.5$ months. CPAP compliance was recorded after 3 months, but not at 12-month follow-up.

\section{Effect of CPAP treatment on arterial stiffness}

No significant change from baseline was observed in cfPWV after CPAP treatment for an average of 10.5 months, delta cfPWV (follow-up - baseline): $-0.1 \mathrm{~m} / \mathrm{s}, 95 \% \mathrm{CI}-0.6,0.4, \mathrm{P}=$ 0.6 , for the group as a whole (Table 3 ).

We observed no significant difference in the change in cf- 
Table 1. Baseline Characteristics

\begin{tabular}{|c|c|}
\hline $\mathrm{N}$ & 46 \\
\hline Age (years) & $63.8 \pm 6.5$ \\
\hline Male, $\mathrm{n}$ & $37(80.4 \%)$ \\
\hline Diabetes duration (years) & $16.1 \pm 9.7$ \\
\hline Apnea-hypopnea index (events/h) & $34(15-73)$ \\
\hline Oxygen desaturation index (events/h) & $36.4 \pm 15.7$ \\
\hline BMI $\left(\mathrm{kg} / \mathrm{m}^{2}\right)$ & $34.7 \pm 3.9$ \\
\hline Systolic blood pressure (mm Hg) & $136.4 \pm 12.9$ \\
\hline Diastolic blood pressure (mm Hg) & $80.4 \pm 6.9$ \\
\hline $\mathrm{HbA1c}(\mathrm{mmol} / \mathrm{mol})$ & $64.7(53-87)$ \\
\hline Fasting blood glucose (mmol/L) & $9.5 \pm 2.3$ \\
\hline Creatinine $(\mu \mathrm{mol} / \mathrm{L})$ & $83.3(43-203)$ \\
\hline Total cholesterol (mmol/L) & $4.0 \pm 0.9$ \\
\hline HDL cholesterol (mmol/L) & $1.2 \pm 0.3$ \\
\hline LDL cholesterol (mmol/L) & $2.1 \pm 0.8$ \\
\hline Triglyceride (mmol/L) & $1.9(0.6-3.9)$ \\
\hline \multicolumn{2}{|l|}{ Smoking } \\
\hline Currently, n (\%) & $6(13.0 \%)$ \\
\hline Former, n (\%) & $18(39.1 \%)$ \\
\hline Never, n (\%) & $22(47.8 \%)$ \\
\hline Statin use, $\mathrm{n}(\%)$ & $36(78.3 \%)$ \\
\hline Aspirin use, n (\%) & $25(54.4 \%)$ \\
\hline \multicolumn{2}{|l|}{ Antidiabetic medication } \\
\hline No medication, $\mathrm{n}(\%)$ & $3(6.5 \%)$ \\
\hline Metformin, n (\%) & $36(78.3 \%)$ \\
\hline Insulin, n (\%) & $29(66.0 \%)$ \\
\hline GLP-1 analog, n (\%) & $27(58.7 \%)$ \\
\hline DPP-4 inhibitor, n (\%) & $3(6.5 \%)$ \\
\hline SGLT2 inhibitor, n (\%) & $5(10.9 \%)$ \\
\hline Sulphonylurea, n (\%) & $1(2.2 \%)$ \\
\hline \multicolumn{2}{|l|}{ Blood pressure medication } \\
\hline No medication, $\mathrm{n}(\%)$ & $4(8.7 \%)$ \\
\hline Beta blockers, n (\%) & $18(39.1 \%)$ \\
\hline Thiazide, n (\%) & $19(41.3 \%)$ \\
\hline ACE-inhibitor, n (\%) & $24(52.2 \%)$ \\
\hline AT2 antagonist, n (\%) & $14(30.4 \%)$ \\
\hline Calcium blockers, n (\%) & $18(39.1 \%)$ \\
\hline Loop diuretics, n (\%) & $8(17.4 \%)$ \\
\hline Other, n $(\%)$ & $7(15.2 \%)$ \\
\hline cfPWV (m/s) & $10.7 \pm 2.0$ \\
\hline
\end{tabular}

BMI: body mass index; HbA1c: hemoglobin A1c; HDL: high-density lipoprotein; LDL: low-density lipoprotein; GLP-1: glucagon-like peptide; DPP-4: dipeptidyl peptidase 4; SGLT2: sodium-glucose cotransporter 2; ACE: angiotensin-converting enzyme; AT2: angiotensin-2; cfPWV: carotid-femoral pulse wave velocity.
Table 2. Multivariate Analysis With Baseline cfPWV as Dependent Variable

\begin{tabular}{llll}
\hline Independent variables & Coefficient & $\mathbf{P}$ & $\mathbf{9 5 \%}$ CI \\
\hline Sex $($ male $)$ & -0.26 & 0.45 & $-0.96,0.43$ \\
AHI $(5$ events $/ \mathrm{h})$ & 0.04 & 0.41 & $-0.05,0.13$ \\
HbA1c $(5 \mathrm{mmol} / \mathrm{mol})$ & 0.29 & 0.001 & $0.12,0.47$ \\
Age $(5$ years $)$ & 0.47 & $<0.001$ & $0.25,0.68$ \\
BMI $\left(5 \mathrm{~kg} / \mathrm{m}^{2}\right)$ & 0.1 & 0.67 & $-0.35,0.55$ \\
Diabetes duration $(5$ years $)$ & 0.19 & 0.01 & $0.04,0.33$ \\
Systolic BP $(5 \mathrm{~mm} \mathrm{Hg})$ & 0.09 & 0.12 & $-0.02,0.19$ \\
Creatinine $(5 \mu \mathrm{mol} / \mathrm{L})$ & -0.08 & 0.01 & $-0.14,-0.02$ \\
Smoking & 1.1 & 0.01 & $0.26,1.93$ \\
\hline
\end{tabular}

cfPWV: carotid-femoral pulse wave velocity; AHI: apnea-hypopnea index; BMI: body mass index; HbA1c: hemoglobin A1c; BP: blood pressure; $\mathrm{Cl}$ : confidence interval.

PWV, between the patients who had been treated for 9 months and patients who had received 12 months of treatment with CPAP, delta cfPWV 12 months - delta cfPWV 9 months: -0.25 $\mathrm{m} / \mathrm{s} ; 95 \%$ CI $-1.3,0.8, \mathrm{P}=0.6$.

Adjusting for mean arterial pressure (MAP), heart rate (HR), number of antihypertensive drugs and BMI did not alter the above findings (results not shown).

\section{Effect of CPAP treatment on BP}

No significant change from baseline was observed in systolic $\mathrm{BP}$ at follow-up (follow-up - baseline): $1.6 \mathrm{~mm} \mathrm{Hg}, 95 \% \mathrm{CI}$ : $-2.27,5.46, \mathrm{P}=0.42$ for the group as a whole (Table 3 ).

We observed no significant difference in the change in systolic BP between the patients who had been treated for 9 months and patients who had received 12 months of treatment with CPAP, delta BP 12 months - delta BP 9 months: $-6.8 \mathrm{~mm}$ Hg, 95\% CI: $-14.2,0.5, \mathrm{P}=0.1$.

After adjusting for number of antihypertensive drugs and BMI the results remained unchanged (results not shown).

\section{Discussion}

In the current study, we observed no effect of prolonging CPAP treatment from 3 months to a median of 10.5 months on cfPWV or BP.

Both type 2 diabetes and OSA are associated with in-

Table 3. Change in Arterial Stiffness and Blood Pressure at Follow-Up

\begin{tabular}{lllll} 
& Baseline & At follow-up & Change & P \\
\hline cfPWV (m/s) & 10.7 & 10.6 & -0.12 & 0.64 \\
Systolic BP (mm Hg) & 136.4 & 138.1 & 1.60 & 0.42 \\
Diastolic BP (mm Hg) & 80.4 & 79.8 & -0.58 & 0.57 \\
\hline
\end{tabular}

cfPWV: carotid-femoral pulse wave velocity; BP: blood pressure. 
creased BP, arterial stiffness and excess mortality $[4,5,11,13]$. Several studies have previously shown that CPAP treatment of OSA can reduce arterial stiffness and BP in various patient populations [17-19]. However, none of these studies have been conducted in a population of patients all of whom had type 2 diabetes. Furthermore, most of the studies have been of short duration. In our original randomized study [15] we observed no effect of CPAP treatment on arterial stiffness or BP after 3-month treatment with CPAP. We speculated that the lack of effect could be attributed to the short duration of treatment. However, the results of our current study show that extending the duration of treatment does not reduce arterial stiffness or BP in our population of patients with OSA and type 2 diabetes. It is noteworthy that despite a wide range of baseline AHI, the severity of OSA had no independent influence on baseline cfP$\mathrm{WV}$, indicating that intervention with CPAP a priori is unlikely to change cfPWV in patients with OSA and type 2 diabetes.

The lack of change in arterial stiffness observed in our study, is in agreement with recent findings by Galerneau et al [20], who found no reduction in arterial stiffness after treatment with CPAP for a median of 7.5 years in obese patients with OSA, of whom $20 \%$ had diabetes. These results were found in spite of high adherence to CPAP treatment. Likewise, no effect on BP was observed.

The lack of effect observed in our study can probably in part be contributed to our study population. It has previously been shown that the increased arterial stiffness in patients with OSA is primarily caused by conventional risk factors for $\mathrm{CV}$ disease such as age, BP and diabetes whereas the severity of OSA is only a minor contributor [21].

We included a population of patients with type 2 diabetes. At baseline, our patients had a high prevalence of hypertension, high BMI and long duration of diabetes. We showed that age, diabetes duration and poor glycemic regulation all are associated with increased arterial stiffness. The fact that diabetes is a major contributor to arterial stiffness has been shown by other study of Laugesen et al [22], and by the findings of Galerneau et al [20], who showed a $13.75 \%$ increase in PWV in patients with diabetes compared to patients without diabetes. Similar, a $12.63 \%$ increase was found in patients with hypertension compared to normotensive individuals. The presence of diabetes and hypertension may cause vascular changes, which cannot be reversed with CPAP treatment.

Diagnosing OSA and treatment with CPAP in patients with diabetes should still be prioritized as CPAP has been shown to reduce daytime sleepiness and improve quality of life [23]. However, our study shows that the indication for CPAP treatment should not be to reduce arterial stiffness, at least not in patients with long duration of diabetes. Further studies should focus on treating OSA in patients with newly diagnosed diabetes to see if early intervention can reduce progression of arterial stiffness in these patients.

Our study has several strengths; we used the gold standard for evaluating arterial stiffness. We had a longer follow-up time than most studies allowing us to evaluate the effects of CPAP treatment over a longer period of time. We examined the effects of CPAP in a homogeneous population of patients with diabetes, which is important because of the high degree of co-existence of OSA, and diabetes.
Our study also has several limitations. The major limitation of our study is the lack of a control group after the initial 3 months. We observed no change in arterial stiffness in our CPAP treated patients, but cannot exclude the possibility that the intervention may have prevented a potential increase in arterial stiffness in untreated patients. However, the findings by Galerneau et al [20] showed that compliance to CPAP had no effect on progression of arterial stiffness, therefore it is probably unlikely that we would have observed a difference between treated and untreated individuals. Another limitation is the lack of control over the participant's medication. After the initial 3 months of the study, changes in antidiabetic and antihypertensive treatment were allowed if the patients usual care provider found it necessary. Changes in medication may therefore have influenced the results. However any change in medication is likely to improve BP or diabetes control. Therefore, changes in medication would have been expected to increase any positive effects of CPAP treatment. Thus, changes in medication are unlikely to have altered our results.

We do not have data on CPAP compliance after 12 months. This lack of data prevents us from detecting any effects in patients compliant to CPAP treatment. However, in the study by Galerneau et al [20], CPAP compliance had no significant impact on arterial stiffness. Likewise, in our original randomized study, we saw no difference in arterial stiffness between the compliant and non-compliant patients. Therefore, the addition of compliance data is probably unlikely to alter our findings.

Another limitation is the number of patients accepting participation in the follow-up. Only $64 \%$ of the participants from our original study accepted participation, which potentially could influence our findings. However, we observed no baseline differences between the patients who accepted and those who rejected participation. It is probably unlikely that patients rejecting participation would be highly compliant and have a large effect of CPAP. On the contrary, we would expect that the patients with the greatest subjective effect of the treatment would be most compliant to treatment and most likely to participate at follow-up [24]. These patients could therefore be expected to have the greatest effect of CPAP treatment. Therefore, it is less likely that the results of our study would have shown an effect of CPAP treatment even if all patients had participated in the follow-up.

In our study we found no effect of 9 - 12 months of CPAP treatment on arterial stiffness or BP in overweight patients with long duration of type 2 diabetes and OSA.

\section{Supplementary Material}

Suppl 1. Difference in baseline characteristics of patients accepting participation in the follow-up study and those rejecting participation.

\section{Acknowledgments}

The authors would like to thank study nurses Pernille BanckPetersen, Charlotte Hansen and Tove Larsen at Nordsjaellands 
Hospital for their help in screening patients for OSA. Medical laboratory assistants Lizette Nislev, Lotte Pietraszek and Susanne Mansson at Nordsjaellands Hospital are thanked for their help in conducting measurements on included patients. Nurse Mette Bjerre Damgard, Sleep Disorders Clinic, Elective Surgery Centre, Regional Hospital Silkeborg are thanked for helping with care of CPAP treated patients. I.E.M. GmbH is acknowledged for lending unrestricted access to Mobil-Ograph devices for this study. Maribo Medico is acknowledged for lending unrestricted access to CPAP devices used for treating the patients in the study.

\section{Financial Disclosure}

This study is supported financially by: University of Aarhus, Regional Hospital Silkeborg, the Health Research Fund of the Central Denmark Region, the Danish Diabetes Academy supported by the Novo Nordisk Foundation, Toyota-Fonden, Denmark and Regional Hospital Central Jutland Research Fund.

\section{Conflict of Interest}

None of the authors have declared any conflict of interest. I.E.M. and Maribo Medico have not had any influence on the design or conducting of the study, nor have they influenced the analysis of data or the manuscript.

\section{Informed Consent}

Written consents from all participants were obtained prior to participation.

\section{Author Contributions}

CK performed measurements, collected data, performed statistical analysis and wrote the manuscript. AMB performed measurements, collected data and reviewed/edited the manuscript. PLP, MGK, BT and LT contributed to the discussion and reviewed/edited the manuscript. KWH and EL contributed to the discussion, reviewed/edited the manuscript, and are the guarantors of the study.

\section{Data Availability}

The data supporting the findings of this study are available from the corresponding author upon reasonable request.

\section{References}

1. Storgaard H, Mortensen B, Almdal T, Laub M, Tarnow L. At least one in three people with Type 2 diabetes mellitus referred to a diabetes centre has symptomatic obstructive sleep apnoea. Diabet Med. 2014;31(11):1460-1467.

2. Franco OH, Steyerberg EW, Hu FB, Mackenbach J, Nusselder W. Associations of diabetes mellitus with total life expectancy and life expectancy with and without cardiovascular disease. Arch Intern Med. 2007;167(11):1145-1151.

3. Taylor KS, Heneghan CJ, Farmer AJ, Fuller AM, Adler AI, Aronson JK, Stevens RJ. All-cause and cardiovascular mortality in middle-aged people with type 2 diabetes compared with people without diabetes in a large U.K. primary care database. Diabetes Care. 2013;36(8):23662371.

4. Young T, Finn L, Peppard PE, Szklo-Coxe M, Austin D, Nieto FJ, Stubbs R, et al. Sleep disordered breathing and mortality: eighteen-year follow-up of the Wisconsin sleep cohort. Sleep. 2008;31(8):1071-1078.

5. Gottlieb DJ, Yenokyan G, Newman AB, O'Connor GT, Punjabi NM, Quan SF, Redline S, et al. Prospective study of obstructive sleep apnea and incident coronary heart disease and heart failure: the sleep heart health study. Circulation. 2010;122(4):352-360.

6. Laurent S, Cockcroft J, Van Bortel L, Boutouyrie P, Giannattasio C, Hayoz D, Pannier B, et al. Expert consensus document on arterial stiffness: methodological issues and clinical applications. Eur Heart J. 2006;27(21):25882605.

7. Mitchell GF, Hwang SJ, Vasan RS, Larson MG, Pencina MJ, Hamburg NM, Vita JA, et al. Arterial stiffness and cardiovascular events: the Framingham Heart Study. Circulation. 2010;121(4):505-511.

8. Guerin AP, Blacher J, Pannier B, Marchais SJ, Safar ME, London GM. Impact of aortic stiffness attenuation on survival of patients in end-stage renal failure. Circulation. 2001;103(7):987-992.

9. Upadhya B, Pajewski NM, Rocco MV, Hundley WG, Aurigemma G, Hamilton CA, Bates JT, et al. Effect of intensive blood pressure control on aortic stiffness in the SPRINT-HEART. Hypertension. 2021;77(5):1571-1580.

10. Van Bortel LM, Laurent S, Boutouyrie P, Chowienczyk P, Cruickshank JK, De Backer T, Filipovsky J, et al. Expert consensus document on the measurement of aortic stiffness in daily practice using carotid-femoral pulse wave velocity. J Hypertens. 2012;30(3):445-448.

11. Wahlqvist ML, Lo CS, Myers KA, Simpson RW, Simpson JM. Putative determinants of arterial wall compliance in NIDDM. Diabetes Care. 1988;11(10):787-790.

12. Lehmann ED, Gosling RG, Sonksen PH. Arterial wall compliance in diabetes. Diabet Med. 1992;9(2):114-119.

13. Drager LF, Bortolotto LA, Figueiredo AC, Krieger EM, Lorenzi GF. Effects of continuous positive airway pressure on early signs of atherosclerosis in obstructive sleep apnea. Am J Respir Crit Care Med. 2007;176(7):706-712.

14. Cortuk M, Akyol S, Baykan AO, Kiraz K, Ucar H, Cayli M, Kandis H. Aortic stiffness increases in proportion to the severity of apnoea-hypopnea index in patients with obstructive sleep apnoea syndrome. Clin Respir J. 2016;10(4):455-461.

15. Krogager C, Banghoj AM, Poulsen PL, Kirkegaard MG, Thorsteinsson B, Tarnow L, Hansen KW, et al. Effect of 12 weeks continuous positive airway pressure on day 
and night arterial stiffness and blood pressure in patients with type 2 diabetes and obstructive sleep apnea: A randomized controlled trial. J Sleep Res. 2020;29(4):e12978.

16. Chiu YC, Arand PW, Shroff SG, Feldman T, Carroll JD. Determination of pulse wave velocities with computerized algorithms. Am Heart J. 1991;121(5):1460-1470.

17. Vlachantoni IT, Dikaiakou E, Antonopoulos CN, Stefanadis C, Daskalopoulou SS, Petridou ET. Effects of continuous positive airway pressure (CPAP) treatment for obstructive sleep apnea in arterial stiffness: a meta-analysis. Sleep Med Rev. 2013;17(1):19-28.

18. Lin X, Chen G, Qi J, Chen X, Zhao J, Lin Q. Effect of continuous positive airway pressure on arterial stiffness in patients with obstructive sleep apnea and hypertension: a meta-analysis. Eur Arch Otorhinolaryngol. 2016;273(12):4081-4088.

19. Kohler M, Pepperell JC, Casadei B, Craig S, Crosthwaite N, Stradling JR, Davies RJ. CPAP and measures of cardiovascular risk in males with OSAS. Eur Respir J. 2008;32(6):1488-1496.

20. Galerneau LM, Bailly S, Borel JC, Jullian-Desayes I, Joyeux-Faure M, Benmerad M, Bonsignore MR, et al. Long- term variations of arterial stiffness in patients with obesity and obstructive sleep apnea treated with continuous positive airway pressure. PLoS One. 2020;15(8):e0236667.

21. Saito T, Saito T, Sugiyama S, Asai K, Yasutake M, Mizuno K. Effects of long-term treatment for obstructive sleep apnea on pulse wave velocity. Hypertens Res. 2010;33(8):844-849.

22. Laugesen E, Hoyem P, Stausbol-Gron B, Mikkelsen A, Thrysoe S, Erlandsen M, Christiansen JS, et al. Carotidfemoral pulse wave velocity is associated with cerebral white matter lesions in type 2 diabetes. Diabetes Care. 2013;36(3):722-728.

23. Siccoli MM, Pepperell JC, Kohler M, Craig SE, Davies RJ, Stradling JR. Effects of continuous positive airway pressure on quality of life in patients with moderate to severe obstructive sleep apnea: data from a randomized controlled trial. Sleep. 2008;31(11):1551-1558.

24. Jacobsen AR, Eriksen F, Hansen RW, Erlandsen M, Thorup L, Damgard MB, Kirkegaard MG, et al. Determinants for adherence to continuous positive airway pressure therapy in obstructive sleep apnea. PLoS One. 2017;12(12):e0189614. 